\title{
Thermography as Method of Paraclinic Diagnosis in Diabetic Polyneuropathy
}

\author{
DOREL FIRESCU1 , RADU ANDY SASCAU2*, GHEORGHE RAFTU³, CRISTIAN STATESCU2*, TUDOR CUCIUREANU2*, \\ DAN IULIAN CUCIUREANU 4 \\ ${ }^{1}$ Sf.Apostol Andrei Emergency Hospital, General Surgery II, 177 Brailei Str., 800578, Galati, Romania \\ ${ }^{2}$ Grigore T.Popa University of Medicine and Pharmacy, Faculty of Medicine, I-st Medical Department, 16 Universitatii Str., 700115, \\ lasi, Romania \\ ${ }^{3}$ Ovidius University of Constanta, Faculty of Medicine, 3rd Medical Department, 124 Mamaia Blvd., 900527, Constanta, Romania \\ ${ }^{4}$ Grigore T.Popa University of Medicine and Pharmacy, Faculty of Medicine,3rd Medical Department, 16 Universitatii Str., 700115, \\ lasi, Romania
}

\begin{abstract}
The concept of polyneuropathy comprises the etiological diversity of neurological syndromes characterized by the formation of bilateral and symmetrical lesions of limb peripheral nerves; symmetrical distal sensorymotor polyneuropathy is the most common form of diabetic neuropathy; it is the most frequent chronic complication of diabetes and more than half of the patients make this complication throughout their lives, both pathogenetic mechanisms and epidemiology, its diagnosis and treatment generate many controversy and challenges for both researchers and practitioners. The study batch consisted of 40 diabetic patients admitted to the Neurology Clinic of the Clinical Recovery Hospital and the Diabetes Clinic between January 2014 and December 2018 who were diagnosed according to clinical and electrophysiological criteria, the etiology of which was type II diabetes with a history of more than 5 years of development and who were examined the lower limb skin according to the local protocol. Thermography is already used as a diagnostic and monitoring tool for patients suffering from neuropathies, especially in the case of complex regional pain syndrome (sympathetic reflex dystrophy) and in our case of diabetic polyneuropathy. This non-invasive exploration has great potential for future research on its use in the diagnosis of other neurological diseases involving the peripheral nervous system. Medical imaging analysis provides a useful way to diagnose and monitor multiple physical illnesses; infrared imaging provides information about infrared energy from the surface of the skin. Emission of infrared radiation changes after nerve damage and vascular occlusions.
\end{abstract}

Keywords: diabetic polyneuropathy, peripheral nerves, diabetic neuropathy, thermography

Unbalanced diabetes mellitus produces, through different mechanisms, altered nerve conduction in the central and peripheral nervous system. Pathological changes of the peripheral nervous system, commonly referred to as diabetic neuropathy, are classified by Dyck et al., in: distal symmetric polyneuropathy with sensory, vegetative and motor involvement; proximal symmetric neuropathy; asymmetric focal neuropathy (multiple neuropathies); asymmetric neuropathy combined with distal symmetric polyneuropathy [1,2].

Chronic sensitive-motor symmetric diabetic polyneuropathy (mixed polyneuropathy) starts insidiously at the distal level (fingers) and evolves slowly proximally. It initially affects somatic and autonomous slim fibrous fibres and then the myelinated thick fibres, and in advanced phases motor fibres as well. The affection of the thin sensory fibres causes alteration of thermal and pain perception, and the affection of thick fibres leads to proprioceptive and vibratory sensitivity and osteo-tendon reflexes disorders. Acute sensory polyneuropathy (acute painful polyneuropathy) has a much lower frequency and is characterized by pain of varying but usually intense nature with nocturnal exacerbation. It can be produced or exacerbated by banal stimuli (allodynia). This form of polyneuropathy occurs after long periods of glycaemic imbalance, with significant decreases in weight, or along with the initiation of insulin therapy and has a naturally regressive evolution. The objective neurological changes are minor compared to the intensity of the symptomatology $[3,4]$.
The simplest and most used classification divides diabetic neuropathies into symmetrical and asymmetric. Symmetric variants include autonomic polyneuropathies and neuropathies, asymmetric variants group mononeuropathies, multiplex mononeuropathies, plexopathies, entrapmentsyndromes, radiculopathies and cranial neuropathies $[5,6]$.

The pathology of diabetic neuropathy and its interpretation has been a continuing source of controversy. It cannot yet be asserted that nerve damage is primary or secondary to neural degeneration where demyelination or axonal loss is primary or the main lesion. The pathology of diabetic neuropathy has been described as having a metabolic or ischemic mechanism. What is certain is that diabetes mellitus has the potential to induce pathological changes in cellular and non-cellular components of peripheral nerves [7-9].

Electronic microscopy studies have shown significant loss of non- myelinated fibres. The characteristics of the degenerative changes of these fibres include axon atrophy, accumulation of vesicular elements, and damage to the tubular and filamentary elements of the cytoskeleton. Complete degeneration results in the vaping or denervation of Schwann cell subunits surrounded by basal lamina. Although the low density of the non-myelinated fibres represents the quantitative reflexion of the modified denervation, Schwann cell subunits are considered to be a better indicator in this context [10-12].

The arterial vascularisation of the peripheral nerve trunks (vasa nervorum) is made up of the endoneurial intrinsic

*emails: radu.sascau@umfiasi.ro; cstatescu@gmail.com; tudor.cuciureanu@umfiasi.ro 
vessels and extrinsic vessels at the epinerve and perinerve level. In diabetes, histological changes were described at the level of all vascular components. At the endonerve level, thick-walled vessels and with narrow-lumen calibre were studied closely. Qualitative and quantitative studies have demonstrated endothelial cell hypertrophy and hyperplasia with diminished vascular lumen. Fenestrated endothelial cells, a feature normally present only in the epineural vessels, were seen in the endoneural vessels as a result of the loss of endothelial cell junction. The occlusion of microvascular lumen resulting from endothelial hyperplasia or from fibrin plugs was investigated without confirmation of the studies performed [13-15].

Epineural capillary abnormalities include hyperplasia and basal lamellar thickening. The intima of epineural arterioles is thickened in diabetic neuropathy. However, despite these changes, endoneural micro-vessels are much more affected than the epineural ones with basal lamina thickening, endothelial cell hypertrophy and lumen narrowing [16- 18].

In the average of the denerved arterioles, structural changes have been reported, such as glycogen growth, smooth muscle cell edema, cell detritus accumulation, collagen deposits.

As for the etiopathogenesis of diabetic neuropathy in the research literature, several theories are issued: vascular, metabolic, which may be interfere with other pathogenic mechanisms $[19,20]$.

These mechanisms explain the development of microvascular complications related to plasma glucose levels and the possibility of preventing them by maintaining a near-euglycaemic level in the long term. Metabolic lesions affect small vessels, the so-called vasa nervorum, the vessels that nourish the peripheral nerves. The longer these nerves (namely those from the lower limbs), the greater the risk of being affected, and therefore, typical diabetic neuropathy occurs especially in the lower limbs [21, 22].

On the one hand, this lesion is angiopathic, and on the other hand there are metabolic deviations. Because of the metabolic imbalance, an increased intracellular glucose concentration occurs in a non-insulin dependent pathway; the higher the concentration of glucose in the extracellular medium, the more intracellularly it penetrates [23- 25].

Excess glucose is metabolized in sorbitol and then fructose. In the diabetic, excess sorbitol accumulates in the basal membrane of the capillaries by altering the vasa nervorum resulting in a microangiopathy with consecutive hypoxia.

Excessive activation of aldol reductase induces a deficiency of myoinositol in Schwann cells byaltering nerve conduction.

Oxidative stress and abnormal glycosylation of structural proteins are also known pathogenic mechanisms.

Current studies address other therapeutic targets: neurotrophic factors, insulin receptors for advanced glycation compounds (AGE), phenotypic sodium channel switching, Poly (ADP-ribose) polymerase (PARP).

Subjective sensitivity disorders represent distal paraesthesia, initially in the lower limbs, then including the upper limbs; sometimes pain, more or less violent, with a feeling of constriction or burning occurs [26-28].

Motor disorders usually occur later in the course of the disease, it also starts initially at the lower limbs, symmetrically, with the paresis of the antero-external area of the calves and stepped walking; more rarely the deficit is primarily interested in the posterior area of the calves or thighs [29-31].
Trophic disturbances are frequent and early, manifested by dry skin, squamous, changes with hair loss, ribbed nails, breakable nails.

Vegetative disorders consist of: vasomotor disorders of intestinal motility, hyper or hypo-transpiration, hypersalivation, orthostatic hypotension, Argyl-Robertson sign, etc.

Cranial nerve lesions are rare in diabetic neuropathy, occurring only in cases with an important metabolic imbalance and affecting ocular motricity and the optic nerve with predilection [32-34].

Polyneuropathy is characterized by distal, symmetrical and bilateral distribution of manifestations of interest initially for the lower and then upper limbs and early loss of superficial or profound sensitivity. With the progression of the disease, there is a particular muscular impairment in the small muscles of hands and feet.

The first symptoms that appear are paraesthesia then associated with pain, which has different characters: deaf, startling, tenacious, nocturnal, sometimes becoming a real cause of disability. Usually occurring as bilateral manifestations, symptoms include numbness, paraesthesia, burning sensation, feeling of vibration in the legs, tingling sensation, severe hyperesthesia and pain.

In diabetic patients, due to the collagen glycation process, there is a progressive decrease in the elasticity of collagen-containing tissues; this change is manifested by limitation in joint mobility due to thickening of skin and periarticular structures; there is a flexion deformation of the hand called cheiroarthropathy, which is recognized by the so-called sign of prayer.

A particular condition of lower limb neuropathy is that of the painful - painless foot in which, in addition to pain and paraesthesia mentioned by the patient, there is a decrease in the sensitivity to pain and of the proprioception one at the objective examination $[35,36]$.

A feature of the peripheral arterial involvement in diabetic patients is the frequent association of medium and large artery calcification (mediosclerosis or Monckeberg disease), which is attributed to the affection of the sympathetic nervous system, similar changes being observed in sympatheticomized patients.

Foot ulceration caused by diabetic neuropathy, trauma, and peripheral vascular disease may result in a lifethreatening infection or endanger the integrity of the affected limb; foot infections are common in patients with diabetes and are associated with high morbidity and increased risk of lower limb amputation.

Knowing the causes and mechanisms that increase the risk of foot injuries and then their production in patients with diabetes is indispensable for the effective application of prevention and treatment measures. The diabetic foot injury occurs as a consequence of the interaction of several factors [37- 39].

Charcot arthropathy normally causes obvious foot deformations, thus severely affecting its functionality, causing unusually large pressure loads during walking. The presence of calluses is a predictive element of the occurrence of ulceration.

Mobility of the joint is defined as the movement of that joint, and is related to age, gender, and ethnic origin; limitation of joint mobility in the foot and ankle in diabetic patients leads to an increase in planting pressure, being closely related to the occurrence of ulceration with the same localization.

In the pathophysiology of diabetic footulceration, there are multiple mechanisms, including neuropathy, peripheral vascular disease, foot deformities, the presence of 
abnormal pressures and long duration of diabetes evolution [40- 42].

Diabetic foot is a concept, a working tool designed to draw attention to the need for an integrated approach to the causes and management of one of the most common, expensive and invalid complications of diabetes, lower limb amputations.

Lower limb obliteration arteriopathy is the most common form of peripheral vascular affection in patients with diabetes mellitus. This consists of the progressive reduction of blood flow in the lower limb arteries due to the progressive narrowing of their lumen produced by atherosclerotic plaques. In pathogenic arteriopathy four pathogenic processes occur: atherogenesis, arterial thrombosis, arterial embolism and arterial spasm.

The main lesion in obliterative arteriopathy is atheroma or atheromatous plaque which consists mainly of cellular diffusion with various lipids and especially cholesterol deposited in the intimal layer of the artery [43- 45].

The most characteristic symptom of chronic and progressive arterial obstruction of the lower limbs is intermittent claudication, that is, a pain at the level of the buttock, thigh, calve or leg that occurs while walking and disappears within a maximum of 10 min of stopping the effort.

Polyneuropathies are a group of diseases that occur by including in a pathological process the nerve endings that are furthest from the spinal cord, practically the nerve endings distributed to the muscles of the fingers of the anatomical hands and feet are affected.

The clinical examination includes: palpation of the arterial trunk allows at the same time to highlight different parietal indurations, different sinuosities and even arterial aneurysms; the calculation of the systolic index has value in the diagnosis of some arteritis; the Rotschow posture test is appreciated by many authors because it is easy to execute and provides data on large arterioles (inaccessible to palpation), the vascular end and collateral circulation; measuring the skin perfusion pressure is a method by which the blood flow is estimated in the ischemic limbs; the Tarfis-Samuel test consists of placing the patient on the back and raising the calves at 65 degrees, then the pedalling movement is performed for $10 \mathrm{~min}$.

During diabetic neuropathy, the electrophysiological exam shows the presence of the eventual signs of denervation in the muscle areas that may be affected by polyneuropathy. These consist of spontaneous denervation activity (fibrillation and slow denervation potentials) and debilitation of the voluntary route (neurogenic deficit index).

Medical thermography is unique in its ability to visualize some of the physiological and metabolic processes that take place in the human body; it is the method of determining skin temperature that consists in capturing skin images with an infrared-sensitive film and is based on the fact that warm objects emit infrared radiation. The warmer a body is, the more it emits a greater amount of infrared radiation.

Through the thermographic method, the temperature is monitored and recorded allowing thus the thermal flow visualization. There are three types of thermography: Liquid Crystal Thermography (LCT); Infrared Thermography (IRT); Microwave Thermography MWT) [3, 4].

Thermography has been successfully used in detecting and characterizing the following neurological disorders: irritation and compression of nerve roots, peripheral nerve lesions, and occlusive diseases of cerebral vessels, migraine, spinal cord injuries and pain syndrome differentiation.
The advantageous features of thermography (painless, non-invasive, without adverse biological effects, is objective, produces a dynamic recording) make it suitable for characterizing neurological dysfunctions.

Diabetic neuropathy affects lower limbs much more frequently than other parts of the body $[7,8]$. The thermography applied in diabetic foot monitoring provides useful information about skin temperature and peripheral vascularisation. The thermographic method as a method of paraclinical diagnosis in diabetic polyneuropathy in current practice.

\section{Experimental part}

\section{Material and method}

The study batch consisted of 40 diabetic patients admitted to the Neurology Clinic of the Clinical Recovery Hospital and Diabetes Clinic between J anuary 2014 and December 2018 that were investigated by means of the cutaneous thermography that completed the usual algorithm of the investigations.

Thermography provides a picture of temperature variations on the surface of the skin by capturing the infrared radiation emitted by it. The diabetic patient with multiple comorbidities, with subclinical or clinical disorders of the peripheral vascularisation, plantar temperature may show changes in the direction of decrease.

\section{Results and discussions}

Thermographs have been performed on patients with diseases that have successfully allowed to perform thermographic scans on the areas of interest (lower limbs, trunk, upper limbs, cephalic extremity).

Being particularly sensitive to the temperature differences of various parts of the body, the thermograph may show areas of slightly modified temperature, the temperature that from a medical point of view may be the result of an inflammatory or other process.

Thermographic scanning involves capturing images remotely without the subject being touched with any object while the images are captured. First, a general scan will be done, tracking all possible affections. From certain areas highlighted by the thermograph, images are taken and analysed. Afterwards, all the thermograms obtained from scanning are interpreted and a conclusion is reached, establishing the final diagnosis.

Thermography is a method of non-invasive imaging investigation, with no side effects and no contraindications for the patient. Unlike other imaging investigation methods, medical thermography is completely risk-free for the patient and for the thermographer.

It is a diagnostic method by which the temperature differences from the surface of the body are visualized, corresponding to physiological or pathological changes both from the surface ad the depth of the skin. By analysing these temperature differences, a diagnosis can be made; is a contactless, non-invasive, non-irradiating method that can be repeated as many times as needed, being basically a special photo of the body's thermal energy. Temperature gives us information about the physiological processes that are taking place, and these processes are disturbed long before the structural changes of an organ appear. The thermograph captures the infrared light radiation. Infrared radiation is emitted by the warmth of the human body, and the thermograph translates it into an image made of a series of colours from the visible spectrum.

Infrared spectrum radiation is emitted by the heat of the human body. For certain diseases, there are well-defined areas on the surface of the body that change their temperature. There is a difference in temperature as 
compared to neighbouring areas or a certain temperature difference as compared to the normal value. This temperature difference is called the temperature gradient and may indicate a possible affection of sub-tegumentary structures in that area.

Selected patients submitted at the entrance to the study investigations of nerve conduction velocity performed in outpatientfacilities (electriophysiology practices) or during admission to the Clinic of Neurology of the Clinical Recovery Hospital.

Patient selection criteria were: identification of patients suffering from diabetic polyneuropathy, establishment of epidemiological data on gender, average age of symptomatology, living conditions and social context of patients with diabetic polyneuropathy, determination and analysis of variability of clinical manifestations of this pathology; evaluation of the impact of diabetic polyneuropathy on the patient's quality of life, identification of the main pathologies associated with diabetic polyneuropathies and the search for possible correlations in terms of both their etiology and the way of life of the patients included in the study, the analysis of diagnostic possibilities in case of diabetic polyneuropathy; role of thermography in diabetic polyneuropathy.

The study group consisted of 20 diabetic patients admitted to the Neurology Clinic of the Clinical Recovery Hospital and the Diabetes Clinic. The casuistry was divided into two study batches (Batch I - 20 patients with type 2 diabetes and Batch II - 20 patients with type 1 diabetes), depending on the type of diabetes.

The age of the patients ranged from 20 to 81 years, the average of the group being $58.90 \pm 12.37$ years, being homogeneous between the batches analysed.

Compared to study batches, the mean age in men was higher than that observed for women in type 1 diabetics, which is not maintained in the batch of type 2 diabetic patients, but the differences were not statistically significant.

Distribution by place of origin revealed a slightly higher frequency of patients with type 2 diabetes mellitus.

The distribution according to living with someone factor outlined the homogeneity of the study groups, $36.7 \%$ of the patients in the first batch and $40 \%$ of the second batch lived alone.

Of the co-morbidities, we have diabetic retinopathy established in the history of $57.8 \%$ of patients with type 2 diabetes and of $59 \%$ of type 1 diabetics, statistically insignificant distributions from a statistical point of view. Chronic kidney disease was significantly more common in patients with type 2 diabetes. HTA was present in the personal history of $83.6 \%$ of patients with type 2 diabetes and of $80 \%$ of type 1 diabetes. Dyslipidaemia was significantly more common in patients with type 2 diabetes.

In the personal pathological history, limb ulcerations were noted in only $15.3 \%$ of patients with type 2 diabetes with an estimated risk of 1.46 times higher. The presence of signs and symptoms was noted more frequently in the lower limbs in both type 2 diabetes (49.3\%), but especially in those with type 1 diabetes (79\%), statistically significant differences.

The case study showed a burning sensation or numbness in the lower limbs / feet, both in patients with type 2 diabetes (66.2\%) and in those with type 1 diabetes (78\%). We also noted paraesthesia, lower limb weakness, lower limb cramps, lower limb pain $[5,6]$.

From the pathology associated with diabetic neuropathy, type 1 diabetics more frequently noted ulcers present in $19 \%$ of subjects on the left foot and in $21 \%$ on both feet compared to the current incidence of ulcerations in patients with type 2 diabetes of only $15.3 \%$, ulcerations that cause relative risk approximately 2 times higher.

Hyperkeratosis, as a neuropathy associated disorder, did not show significant differences on types of diabetes. The hammer fingers were significantly more common in patients with type 2 diabetes. Amyotrophy did not show significant percentage differences depending on the type of diabetes ( $24.3 \%$ vs. $39 \%$ ).

In the studied case, $80 \%$ of the type 2 diabetic patients and $60 \%$ of the type 1 patients have peripheral pulse in the left limb, which is also noticeable in the right foot with $78.6 \%$ and $59 \%$ respectively, insignificant percentage differences from a statistical point of view.

Achilles reflex in patients with type 2 diabetes remained absent after retest in $37.3 \%$ of patients, both on the left and right feet. In patients with type 1 diabetes, the absence of the Achilles reflex was noted in $21 \%$ of patients.

In the case study, it is noted that approximately $1 / 2$ of the patients $(53 \%)$ recorded nerve conduction velocity below $43 \mathrm{~m} / \mathrm{s}$ on the sural nerve and below $39 \mathrm{~m} / \mathrm{s}$ on the peroneal / tibial nerves.

Thermography in patients with conditions presentat the time of study-by drawing the ROC curve, on the case study studied, it is noted that SPOT, regardless of the tested foot, is a good predictor of neuropathy in patients who also associated ulcerations at the time of study - left foot (AUC $=0.670$; IC95\%: 0.576-0.783) and right foot (AUC = 0.756; IC95\%: 0.661-0.852). SPOT thermography is a good predictor of neuropathy in patients who have associated osteolysis at the time of study; SPOT, regardless of the foot tested, it is a good predictor of neuropathy in patients who have associated hyperkeratosis at the time of study; SPOT thermography is not a good predictor of neuropathy in patients who have associated amyotrophy at the time of study.

SPOT reassessment - in patients in group I, patients with type 2 diabetes, mean SPOT values decreased by $6.7 \%$ in the left foot and increased by $0.3 \%$ in the right foot.

In patients in batch II, patients with type 1 diabetes, mean SPOT values decreased by $37.6 \%$ in the left foot and increased by $1.9 \%$ in the right foot.

A characteristic of diabetic neuropathy is symmetry of symptoms and signs (in both feet), and our results indicate that the etiology in diabetic patients may be mixed, which should be investigated further in clinical practice.

Medical thermography can be considered a step forward in preventive medicine. The term medical thermography refers to a non-invasive investment for the patient.

Thermography has been successfully used in the detection and characterization of the following neurological disorders: irritation and compression of nerve roots, peripheral nerve lesions, occlusive diseases of cerebral vessels, migraine, spinal cord injuries and pain syndrome differentiation.

The advantageous features of thermography (painless, non-invasive, without adverse biological effects, it is objective, produces a dynamic recording and is no more expensive than other diagnostic procedures) makes it suitable for diagnosis of neurological disorders.

In 2002, Saldo - Butkovic et al. carried out a characterization of the thermal models in the case of neurologically normal patients and those who had peripheral neural structures in the lumbosacral region with particular reference to the anterior femoral area. The authors conclude that it was an isothermal imbalance that altered the inclination angle of the isothermal lines relative to the horizontal line and in the presence of isotherm. This may be a clear sign of injury to the peripheral nervous 
system. They proposed introducing this diagnostic criterion into clinical practice.

Thermography can be used to identify a local affection and determine its extent. It is a useful and sensitive technique for detecting local radio-induced conditions, especially in early and latent phases when there are no relevant symptoms.

Infrared thermography is superior in terms of diagnostic possibilities in partial body exposure, especially in the extremities.

In 2003, Stulin et al., published a paper debating the problem of use of thermography in the field of neurology, but they have notcome to any conclusion. Theymentioned, however, the use of thermal imaging in the recognition of pre-cancerous and cancerous conditions in mammary, articular diseases and in the diagnosis of arterial occlusion. In the same year, Carbone et al., published another paper on new possibilities for investigating and detecting arteriosclerotic lesions in carotid arteries, and cited among them intravascular thermography as well.

\section{Conclusions}

One of the types of images that have developed over the last decades is represented by thermal imaging. They are captured by infrared cameras and their development over the past few years has been remarkable; they can indicate small changes in body temperature.

There are studies on the use of thermography in: diagnosis of cancer, diagnosis of muscle damage, diagnosis of ischemia in patients with diabetes and evaluation of muscle recovery.

The study has shown that there is a diabetic foot injury both from the angle of polyneuropathy and the presence of peripheral arterial disease at different stages of evolution, even if clinically the patient had a pulse in the peripheral arteries.

\section{References}

1.***American Diabetes Association (ADA). Diagnosis and Classification of Diabetes Mellitus. Diabetes Care. 2012;35(1):S11. 2.ARGOFF CE, COLE EB, FISHBAIN DA, IRVING GA. Diabetic Peripheral Neuropathic Pain: Clinical and Quality-of-Life Issues, Mayo Clin Proc 2006;81(suppl):S3-S11.

3.CUCIUREANU, D.I., STATESCU, C., SASCAU, R.A., CUCIUREANU, T., CONSTANTINESCU, V.A., HINGANU, D., PREDA, C., HINGANU, M.V., TURLIUC, M.D., Particularities of using contrast agents in diagnosis of stroke. Rev Chim (Bucharest), 70, no.2, 2019, p. 685-688

4.MINDRUTA IR, BAJENARU OA, PANEA CA, ET AL. Experience with lacosamide in treating focal epilepsy patients in Romania: efficacy, safety and time to reach response [abstract no. p332]. Epilepsia. 2014;55(Suppl 2):110.

5.BANDEIRA F, NEVES EB, MOURA MAMD, NOHAMA P. A termografia no apoio ao diagnostico de lesao muscular no esporte. Rev Bras Med Esporte 2014; 20: 59-64.

6.BANDEIRA F, MOURA MAMD, SOUZA MAD, NOHAMA P, NEVES EB. PODE a termografia auxiliar no diagnóstico de lesões musculares em atletas de futebol? Rev Bras Med Esporte 2012; 18: 246-51.

7.BOYKO EJ, AHRONI JH, COHEN V, NELSON KM, HEAGERTY PJ. Prediction of diabetic foot ulcers occurence using commonly avaible clinical information: the Seatle Diabetic Foot Ulcers Study. Diabetes Care 2006;29:1202-1207.

8.BOULTON AJM., The pathway to ulceration. Aetiopathogenesis, in Bouton AJM Cavanagh PR., Rayman G. The foot in diabetes, fourth Edition, J ohn Wiley \&Sons Ltd., 2006, pg 30-41.

9.BOULTON AMJ, VINIK AI, AREZZO JC, BRIL V, FELDMAN EL, FREEMANN R, ET AL.Diabetic neuropathies: a statement by the American Diabetes Association. Diabetes Care. 2005.
10.BOULTON AJM, ARMSTRONG DG, ALBERT SF, FRYKBERG RG, HELLMAN R, LAVERY LA, LEMASTER JW, MILLS JL, MUELLER MJ, SHEEHAN P, WUKICH DK. Comprehensive foot examination and risk assessment: a report of the Task Force of the Foot Care Interest Group of the American Diabetes Association, with endorsment by the American Association of Clinical Endocrinologists. Diabetes Care 2008:3:1679-1685.

11.CUCIUREANU DI, NITA A, CUCIUREANU A, CUCIUREANU T, CONSTANTINESCU IM. Experience with firstepisode of consciousness loss assessment in a regional center of Romania. [abstract no. p638]. Epilepsia. 2016; 57 (suppl. 2): 194

12.BOANGHER S, MESPOUILLE P, GOFFETTE S, VAN PESCH V, CUCIUREANU D. Herpes simplex encephalitis relapse associated with positive $\mathrm{N}$-methyl-d-aspartate receptor antibodies. ACTA NEUROLOGICA BELGICA; 2018; 118(4): 533-535. DOI: 10.1007/s13760018-0897-9

13.BROWNLEE M, The pathobiology of diabetic complications. A unifying mechanism. Diabetes, 2005;54:1615-1625.

14.CARALIS D.G. AND G.L.BAKRIS.,Lower extremity arterial disease.Totowa NJ., Humana Press, 2005.

15.BRODSKY JW. THE DIABETIC FOOT. IN MANN RA, COUGHLIN MJ, SALTZMAN CL (EDS): Surgery of the Foot and Ankle. Philadelphia:Elsevier, 2006.

16.DUCEAC, L.D., LUCA, A.C., MITREA, G., BANU, E.A., CIUHODARU, M.I., CIOMAGA, I., ICHIM, D.L., BACIU, G., Ceftriaxone Intercalated Nanostructures Used to Improve Medical Treatment, Mat.Plast, 55, no.4, 2018, p. 613-615.

17.BOTEZATU, C, DUCEAC, LD, MASTALIER, B, STAFIE, L, JITAREANU, CM, LUCA, AC, TARCA, E, MITREA, G, IORDACHE, AC, PATRASCU, T. Hepatic cystic echinococcosis studied in a family group. INTERNATIONAL JOURNAL OF MEDICAL DENTISTRY, 2018, 22 (4): 346-350.

18.BOWER JH. LEVIN AND O'NEAL'S The Diabetic Foot with CD-ROM, Ed. Elesevier, 2008;46-68.

19.CHAN JCN, MALIK V, JIA W, ET AL. Diabetes in Asia: epidemiology, risk factors, and pathophysiology, JAMA 2009;301:2129-2140.

20.DIETER R.S.,DIETER R.A., Peripheral arerial disease. New York, McGraw-Hill Medical, 2009.

21.DU H, LI BH.,Clinical and neurophysiological features of 700 patients with diabetic peripheral neuropathy. Zhonghua Nei Ke Za Zhi,2005, 44(3), 173-176.

22.DE MEIRA LF, KRUEGER E, NEVES EB, NOHAMA P, DE SOUZA MA.Termografia na Area biomEdica. Pan Am J Med Therm 2014; 1: 3141.

23.EDUARDO BORBA NEVES ET AL, The Open Neurology Journal, 2015, 9, 24-27.

24.FERNANDEZ CUEVAS I. Efecto del entrenamiento de resistencia, velocidad y fuerza en la temperatura de la piel a travEs de la termografla infrarroja. Tesis doctoral. Facultad de Ciencias de la Actividad Física y del Deporte, Universidad Politecnica de Madrid; 2012.

25.FISHER M AND J .J.V. MCMURRAY, Diabetic cardioloy.Chicester, West Sussex, England; Hoboken NJ., J ohn Wiley and Sons, 2007.

26.HEIDRICH H., Are there predictors for the outcome of a PGE1 treatment in peripheral arterial disease with critical limb ischaemia? Vasa 34(2), 2005, 101-107.

27.JUDE EB., Chaecot foot:what's new in pathogenesis and medical management? in Boulton AJ M., Cavanagh PR., Rayman G.The foot in diabetes Fourth edition, J ohn Wiley \&Sons Ltd.England, 2006,pg.265274.

28.J EONG SM, KANG MJ, CHOI HN, KIM JH, KIM JI. Quercetin ameliorates hyperglycemia and dyslipidemia and improves antioxidant status in type 2 diabetic db/db mice. Nutr Res Pract. 2012;6(3):201-207. 29.MOHLER E.R.M.R., J aff and American College of Physicians. Peripheral arterial disease. Philadelphia. American College of Pgysicians, 2008.

30.PANEA C.,Neuropatia diabetica din punct de vedere al neurologului. BMJ , Editia în limba romana 2004, 11 (4), 120-129. 
31.PARK JE, BARBUL A. UNDERSTANDING THE ROLE OF IMMUNE REGULATION IN W OUND HEALING. AM J SURG 2004;187:11S-16S. 32.PELTZ E, SEIFERT F, MAIHOEFNER C. Diagnostic Guidelines for Complex Regional Pain Syndrome. Handchirurgie Mikrochirurgie Plastische Chirurgie 2012; 44: 135-41).

33.POGORELIK PEPI, Bazele termografiei computerizate, Editura Gr.T.Popa, UMF.lasi, 2005;pag 2-20.

34.SAHLI D., SVENSSON M., LIDGREN J ., OJ BRANDT K., ERIKSSON JW. Evaluation of simple non-invazive techniques for assessment of lower extremity arterial disease, Clin Phisiol Funct.Imagingl, 2005;25):129134.

35.S. SIVANANDAM, M. ANBURAJ AN, B. VENKATRAMAN, M. MENAKA, D. SHARATH, Medical thermography: a diagnostic approach for type 2 diabetes based on non-contact infrared thermal imaging, Endocrine (2012) 42:343-351.

36.SPACEK M AND J.VESELKA . claudicazion pain in the left arm of a coronary artery bypass graft patient using crutches:Coronary subclavian steal syndrome-a case report. Int J.Angiol 2010; 19(1): 4142.

37.SPITTELL J.A. PERIPHERAL vascular disease for cardiologists:a clinical appriach Elmsford, NY.,Futura, 2004.

38.SUBRAMANIAM BAGAVATHIAPPAN, THANGAVELU SARAVANAN, Investigation of Peripheral Vascular Disorders Using Thermal Imaging, Posted:06.19.2008; british Journal of Diabetes and Vascular Disease.2008.Sage Publications, Inc. 2008; 8(2):102-104.
39.SUZUKI Y, KOBAYASHI M, KUWABARA K, KAWABE M, KIKUCHI C,FUKUDA M. Skin temperature responses to cold stress in patients with severe motor and intellectual disabilities. Brain Develop 2013; 35: 265-9.

40.SUGANTHI SS, RAMAKRISHNAN S. Analysis of breast thermograms using gabor wavelet anisotropy index. J Med Sys 2014; 38:1-7. 41.TESFAYE S: DIABETIC NEUROPATHY IN BOULTON AJ M, CAVANAGH PR, Rayman G.The diabetic foot in diabetes, fourth Ediion, John Wiley \&Sons Ltd., 2006, pg.30-41.

42.TESFAYE S., CHATURVEDI N., EATON SEM, WITTE D., WARD JD., FULLER I., Vascular risk factors and diabetic neuropathy., N.Engl I Med 2005, 352:341-350.

43.TESFAYE S, BOULTON AJM, DICK PJ, FREEMAN R, HOROWITZ M, KEMPLER P., Diabetic Neuropathies: update on definitions, diagnostic criteria,estimation of severity, and treatments. Diabetes Care. 2010;33(10):2285.

44.TREECE KA, MCFARLANE RM, POUND N, GAME FL, JEFFCOATE WJ. Validation of a system of foot ulcer classification in diabetes mellitus. Diabetic Medicine 2004;21(9):987-991.

45.ZIMNY S., SCHATZ H., PFOHL M., The role of limited joint Mobility in diabetes patients with an at-risk foot. Diabetes Care, 2004, 27:942946.

Manuscript received: 9.10 .2018 